\title{
Recognition Method for Handwritten Digits Based on Improved Chain Code Histogram Feature
}

\author{
Qian You, Xichang Wang, Huaying Zhang, Zhen Sun and Jiang Liu
}

\begin{abstract}
The chain code histogram feature is a simple and effective feature extraction technology. This paper proposes improvements based on Chain Code Histogram $(\mathrm{CCH})$ and its first differential characteristics. According to the first Differential Chain Code Histogram $(\mathrm{DCCH})$, the turning points in the direction are extracted, and the judging method of Direction Turning Point (DTP) is given. We combine $\mathrm{CCH}$ and DTP into a new feature, then handwritten digits of MNIST database are recognized and classified by Support Vector Machine (SVM) classifier. The experimental results proved that the recognition rate of the improved method is not only higher than $\mathrm{CCH}$ and first differential $\mathrm{CCH}$, but also closely to the recognition rate of their combination. Obviously, the new combination reduces the feature dimension, improves the speed of training and recognition.
\end{abstract}

Keywords: Chain code histogram, - Differential chain code $\cdot$ Direction turning point $\bullet$ Handwritten digit recognition $\bullet$ Support vector machine

\section{Introduction}

As computer technology and Internet technology are widely used, the transformation of the traditional text information on paper to digital format is a main trend in the world today. Digits as a kind of universal symbol in the world, are widely used in the systems of bank check, postal service, online marking and so on $[1,2]$. In practical application, digits generally represent accurate data, and its recognition must be in high accuracy. Although the handwritten digit recognition research has been going on for decades, and get delectable achievements, but compared with

\footnotetext{
Q. You $(\bowtie) \cdot$ X.Wang $\bullet$ Z.Sun

School of Management Science and Engineering, Shandong Normal University, Lixia District,

East of Culture Road 88,250014 Jinan, China

e-mail: qianyou77@163.com

H.Zhang • J.Liu

Shandong Research Institute of Data Processing Oumasoft, 250100 Jinan, China
} 
the human ability to recognize there is a certain distance. Therefore, handwritten digit recognition is still significant in research.

Feature extraction technology is an important part of handwritten digit recognition. We must take peoples' different writing habits into account when chose the features. A successful feature must be stable and easy to be distinguished. $\mathrm{CCH}$ is a relatively successful and simple feature extraction technology. Freeman firstly proposed chain code in 1977[3]. The CCH method has been successfully applied to handwritten Devnagari, Gurumukhi, Malayalam, Arabic [4, 5, 6, 7] character recognition, and received high recognition rate. J. Jain et al. achieved accuracy of $96 \%$ by using $\mathrm{CCH}$ and $\mathrm{DCCH}$ [8]. This method needs more storage space while the speed of training and recognition slows down.

In this paper, we have improved the combination feature based on $\mathrm{CCH}$ and DCCH. Firstly, Direction turning point is computed from DCCH. Then we combine it with $\mathrm{CCH}$ into a new feature, describe the judgment of DTP in details. Finally, we estimate the algorithm by contrast experiment. The handwritten digits in this study used are all from MNIST handwritten numeral database.

\section{Feature Extraction}

The chain code Freeman proposed is divided into 4-direction and 8-direction (Fig.1). This method extracts contour shape of the character pattern, and the contour is coded according to the direction change information of each point with the next one. $\mathrm{CCH}$ is the statistical characteristics of chain code. Similarly, DCCH is the statistical characteristics of differential chain code.
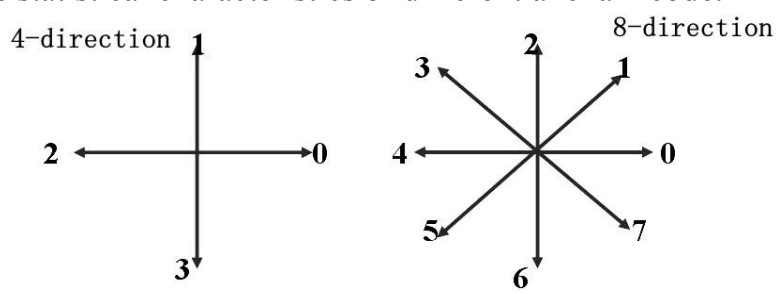

Fig.1 Chain code directions

\subsection{CCH Feature Extraction}

The $\mathrm{CCH}$ feature extraction technology gets directional information from the pixels of the extracted character contour. Subsequently, we count the different codes. The whole process of $\mathrm{CCH}$ feature extraction is showed as follows. 
1) Transform the image into binary format and extract the contour shape of the character pattern.

2) Resize the image to $m * m$, and divide it into $m^{2} / n^{2}$ blocks in the scale of $n * n$.

3) According to traversal sequence of top to bottom and left to right, each contour pixels direction was calculated, and the direction is marked as 8direction as showed in Fig.1.

4) Count the frequency of each direction appears in every block.

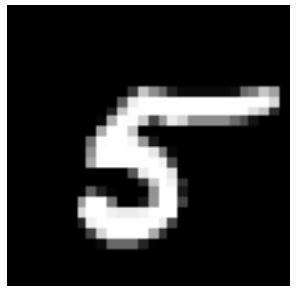

(a)

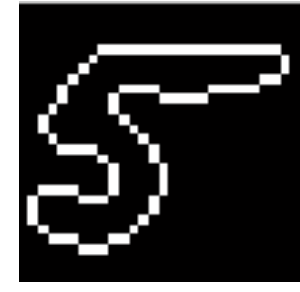

(b)

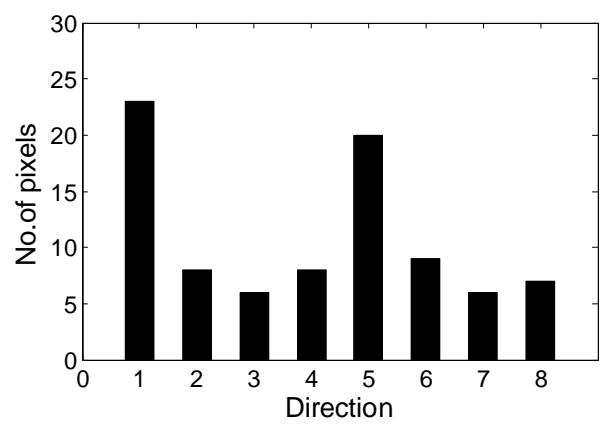

(c)

Fig.2 (a) shows the original image of handwritten digit 5, (b) is the image after preprocessing, and (c) represents the $\mathrm{CCH}$ feature of digit 5

The CCH feature of digit 5 is shown in Fig.2 (C), we use the direction value $1 \sim 8$ instead of $0 \sim 7$ for preventing the gray value 0 of background pixels from mixing up with the direction 0 . The $\mathrm{CCH}$ is the statistical characteristic of the whole character with all blocks.

\subsection{DCCH Feature Extraction}

The $\mathrm{CCH}$ feature only represents the directional information of handwritten digit, without any features on directional change. However, completely different charac- 
ters may have the same characteristics DCCH can solve this problem by providing the variations in the directional information. The first DCCH can be obtained by computing the difference between two neighbor pixels. In general, we use the succeeding one minus the preceding one. The DCCH also can be calculated by counting the steps of the first chain code turning to the succeeding one in clockwise or counterclockwise. For example, a chain code of 4-direction is 10103322, conversely its differential chain code is 3133030 in counterclockwise. There will be not negative values in the differential chain code which obtained through this method.

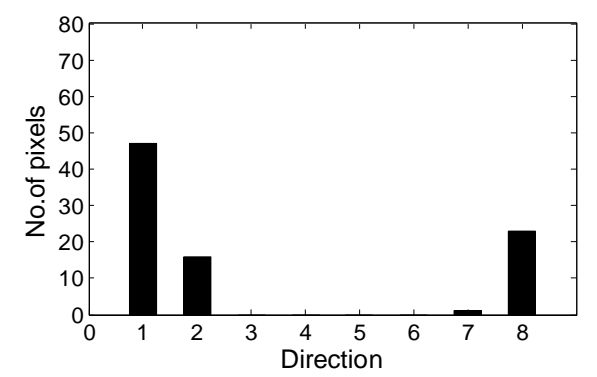

Fig.3 the DCCH representation of digit 5, its original image is fig.2 (a)

The DCCH above is calculated in clockwise. We can notice that the differential chain code concentrating upon 1,2,7,8. Its distribution is in polarized trend. This feature can filter some extra unexpected directional information to some extent.

\section{Modified Feature Extraction Method}

$\mathrm{CCH}$ and $\mathrm{DCCH}$ reflect the direction information and direction change information respectively. If they can be effectively combined, then the combined feature can represent more comprehensive characters. However, their direct combination is bound to cause a problem that the speed of training and recognition becomes slower. To solve this problem, we propose a new feature which is direction turning point (DTP). M. Blumenstein described the distinction of individual line segments [10]. According to this method, we can distinguish the DTP. The distinction of DTP based 8-direction chain code is described in details.

\section{DTP}

The DTP is a point where direction changes sharply. Assuming an 8-direction chain code as $A 1 A 2 \cdots A i A i+1 \cdots A n$, its differential chain code as $B 1 B 2 \cdots B i \cdots B n-1$ 
$(0 \leqslant A i \leqslant 7, \quad \mathrm{i}$ and $\mathrm{n}$ represent nature number, and $1 \leqslant \mathrm{i} \leqslant \mathrm{n})$. So $B i$ is the first differential of $A i$ and $A i+1$. After computing the first differential chain code, if the differential chain code meets any one as follows:

1) $B i \neq 0$, and $B i-1=B i-2=0$;

2) $B i=B i-1=B i-2=1$ or 7 ;

3) $2 \leqslant B i \leqslant 6$.

Then we consider the direction changes sharply at point $\mathrm{Ai}+1$.That is to say, the point $A i+1$ is a DTP. The first condition expresses that the current direction value changes and the previous direction value has sustained more than three times. The second condition represents that the previous direction changed $45^{\circ}$. The last condition indicates that the previous direction changed greater than $90^{\circ}$. Finally, the DTP is marked as 9 in the character pattern. Repeat the operation for the whole differential chain code. So we get the DTP feature of the character image.

In this work, we combined the $\mathrm{CCH}$ feature and the DTP feature. The structure of the combined feature is shown as Table $\mathbf{1}$.

Table 1 The feature vector of each block

\begin{tabular}{ccl}
\hline Frequency of direction 1 & Frequency of direction 2 & Frequency of direction 3 \\
Frequency of direction 4 & Frequency of direction 5 & Frequency of direction 6 \\
Frequency of direction 7 & Frequency of direction 8 & Frequency of DTP \\
\hline
\end{tabular}

\section{The Process of Handwritten Digit Recognition}

In this paper, the process of handwritten digit recognition system is shown in Fig.4. The steps in the system are introduced in following sections.

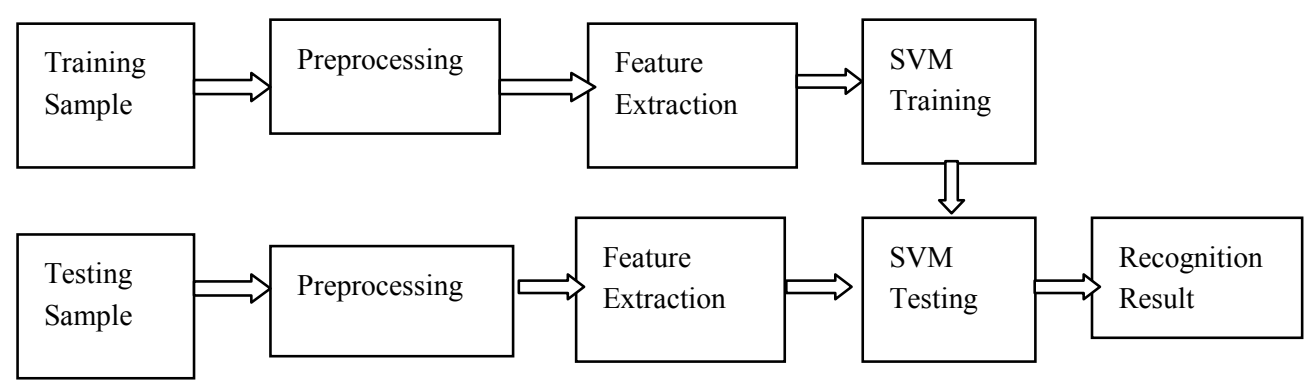

Fig.4 Handwritten numeral recognition system flow chart 


\subsection{Preprocessing}

This part includes image binarization, contour extraction, character position adjustment, and the effect figure after preprocessing as shown in fig.2 (b).We choose $\mathrm{K}$-means clustering algorithm as the binarization methods [9], the clustering centers are 64 and 192. We use canny detector detect the edge of characters. One of the most important is position adjustment, it is illustrated as follows:

1) Find out the width and height of the characters.

2) Shear the image according to the width and height of the characters.

3) Enlarge the sheared image to according to the maximum of the width and height until either of their length is equal to the fixed size.

4) Normalize the image into a fixed size by filling background pixels.

The character position cannot be inclined to one side, the distance from leftmost character to the edge of the image is equal to the right one. So the top distance and the bottom one are.

\section{Extracting feature vector}

Firstly, we capture the $\mathrm{CCH}$ feature. Secondly, DCCH feature is calculated from chain code. Finally, we distinguish DTP based on analysis of first differential chain code. $\mathrm{CCH}$ feature is combined with DTP into a new fusion feature. So the number of vector in each block is 9 .

\subsection{Training and testing}

Supporting Vector Machine has been successfully applied in the field of pattern recognition, such as text recognition, face recognition and so on. It shows good performance in applications [11]. So this part we utilize SVM to train and test. The Radio Basis Function (RBF) is chosen as the kernel.

\section{Experiment Results and Analysis}

In this paper, we improve the combination of $\mathrm{CCH}$ and DCCH by using DTP feature instead of DCCH. In order to demonstrate the effectiveness of this method, all the experiment data are from MNIST handwritten digit image standard database. This database has 60000 training samples and 10000 testing samples. In the expe- 
riment, all the character images are scaled in size of $28 * 28$ and the block size is set to $7 * 7$. Firstly, 60000 training samples and 10000 testing samples are preprocessed, extracted feature vector, and prepared in different required matrixes. Secondly, we gain a classification model by training 60000 samples in the training set. At last, we use the model recognize the 10000 testing samples.

This experiment is conducted in Widows XP operating system and Matlab.R2010.version. To verify the effectiveness of DTP feature, we have done four groups of experiment involves $\mathrm{CCH}, \mathrm{DCCH}, \mathrm{CCH}+\mathrm{DCCH}$ and $\mathrm{CCH}+\mathrm{DTP}$. The penalty parameter $C$ is set to 20 in the experiment. We take the dimensions of feature as the value of $\gamma$.

Table 2 Experiment results of different feature extraction techniques

\begin{tabular}{lllllll}
\hline $\begin{array}{l}\text { Feature } \\
\text { extraction }\end{array}$ & $\gamma$ & $\begin{array}{l}\text { Training } \\
\text { Time (s) }\end{array}$ & $\begin{array}{l}\text { Testing } \\
\text { Time }(\mathrm{s})\end{array}$ & $\begin{array}{l}\text { Model Storage } \\
\text { Space(MB) }\end{array}$ & $\begin{array}{l}\text { No. of } \\
\text { SV }\end{array}$ & $\begin{array}{l}\text { Recognition } \\
\text { Accuracy (\%) }\end{array}$ \\
\hline $\mathrm{CCH}$ & $1 / 128$ & 535 & 245 & 2.84 & 13019 & 97.54 \\
$\mathrm{DCCH}$ & $1 / 128$ & 708 & 321 & 2.63 & 17072 & 95.32 \\
$\mathrm{CCH}+\mathrm{DCCH}$ & $1 / 256$ & 1210 & 553 & 5.35 & 15234 & 98.10 \\
$\mathrm{CCH}+\mathrm{DTP}$ & $1 / 144$ & 587 & 297 & 3.08 & 13600 & 98.00 \\
\hline
\end{tabular}

The performances of handwritten digit recognition based on four feature extraction techniques are shown in Table 2. Obviously, $\mathrm{CCH}$ combined with DCCH achieves the highest recognition rate of $98.00 \%$, CCH combined with DTP falls behind with $0.1 \%$ difference. However, the recognition method based on $\mathrm{CCH}$ and DTP takes shorter time, smaller storage space. Especially the training time of $\mathrm{CCH}$ and DCCH is 1210 s while the training time of CCH and DTP is $587 \mathrm{~s}$. This is almost twice as much. If considered about comprehensive evaluation, $\mathrm{CCH}$ and DTP feature extraction method performs better.

\section{Conclusions and future research}

This paper presented a new fusion feature extraction technique for the recognition of handwritten digit. The new fusion feature is the combination of $\mathrm{CCH}$ and DTP. A recognition system for handwritten digit was developed based on SVM. The new fusion feature achieves a recognition rate of $98 \%$, it is comparable to the direct combination of $\mathrm{CCH}$ and $\mathrm{DCCH}$ of $98.1 \%$. But in the respects of storage space and time-consume, the new fusion feature outperformed the direct combination. It not only expresses the directional information of the handwritten digit but also represents the directional change information. The experiment results reveal that the combined feature of $\mathrm{CCH}$ and DTP is stable and effective. 
In the future research, many improvements may be proposed on preprocessing part, more fusion features will be explored. We may modify out classification technique with more outstanding performance applied to handwritten digit recognition system.

Acknowledgements This study is funded by the Research and Application of High-speed Image Data Acquisition Processing, one of Shandong province Science and Technology Research Program of China (211GZC20106).

\section{References}

1. Hu D (2012). Research and application of handwritten numeral recognition method. SM thesis, University of Nanchang, Nanchang, China.

2. Tong X (2009). Handwritten digital recognition technology research and its application in automatic checking system. SM thesis, Dongbei Normal University, Jilin, China.

3. Freeman H., Davis L.S. (1977). A corner finding algorithm for chain-coded curves. IEEE Transcations on computers , 26(5): 297-303.

4. Singh P., Verma A., Chaudhari N.S. (2011). Performance Evaluation of Classifiers Applying Directional Features for Devnagri Numeral Recognition. Advanced Materials Research, 1042, 403-408.

5. Kaur H., Kumar R. (2011). Slant Correction and Resampling for Online handwritten character Recognition of Gurumukhi Script. SM thesis, Thapar University, India.

6. John J., Pramod K.A., Balakrishnan K. (2011). Offline handwritten Malayalam Character Recognition based on chain code histogram. Emerging Trends in Electrical and Computer Technology (ICETECT), 736-741. doi: 10.1109/ICETECT.2011.5760215.

7. Lawal I.A., Abdel-Aal R.E., Mahmoud S.A. (2010). Recognition of Handwritten Arabic (Indian) Numerals Using Freeman's Chain Codes and Abductive Network Classifiers. Pattern Recognition (ICPR), 1884-1887.

8. Jain J., Sahoo K.S., Prasanna S.R., Reddy G.S.(2012). Modified Chain Code Histogram Feature for Handwritten Character Recognition.C-CSIT. Advances in Computer Science and Information Technology. Networks and Communications, 84, 611-619.

9. Gonzalez R.C., Woods R.E., Eddins S.L.(2004). Digital Image Processing Using MATLAB. Prentice Hall.

10. Blumenstein M., Verma B., Basli H. (2003). A Novel Feature Extraction Technique for the Recognition of Segmented Handwritten Characters. Proceedings of the Seventh International Joint Conference On Document Analysis and Recognition, 1:137-141.

11. Ding S, Qing B, Tan H. (2011). An Overview on Theory and Algorithm of Support Vector Machines. Journal of University of Electronic Science and Technology of China, 40(1):210 . 